\title{
STUDY OF ATTITUDES TOWARDS THE USE OF MOBILE PHONES WHILE DRIVING WITH IMPLICIT ASSOCIATION TESTS AND SELF-ASSESSMENT PROCEDURES
}

\author{
Zhanna Vinogradova \\ Baltic International Academy, Latvia \\ Irina Plotka \\ Baltic International Academy, Latvia \\ Iveta Smirnova \\ Baltic International Academy, Latvia \\ Nina Blumenau \\ Baltic International Academy, Latvia \\ Dmitry Igonin \\ Baltic International Academy, Latvia
}

\begin{abstract}
Analysis of studies shows that in studying attitudes towards risky and safe driving only few researches are based on the use of implicit methods. The aim of the study: the study of attitudes towards the use of mobile phones while driving with the use of Implicit Association Tests and self-assessment procedures. Participants: 69, age 21-59, $M=42, S D=9.02,27$ female and 42 male, all with B category driver licenses, driving experience 9-24 years. Implicit measurements: two experimental procedures of the Implicit Association Test (IAT) developed by the authors for measuring attitudes towards the use of mobile phones while driving: IAT and self-concept IAT Explicit measurements: a self-assessment procedure developed by the authors "Scale of measuring attitudes towards the use of mobile phones while driving," cross-cultural Personality Questionnaire ZKPQ-50-CC (Aluja, Rossier, García, Angleitner, Kuhlman, \& Zuckerman, 2006). A positive relationship between the results of measurements using IAT and self-concept IAT was found in participants with high anxiety. The effect size obtained with the IAT is larger than the effect size obtained with self-concept IAT. A relationship between the results of measurement of attitudes towards the use a mobile phone while driving, measured by experimental procedures and the personal factors was found.
\end{abstract}

Keywords: Attitudes towards driving, drivers, explicit, implicit measurements, personality factors. 
Vinogradova et al., 2018. Study of Attitudes Towards the Use of Mobile Phones While Driving with Implicit Association Tests and Self-Assessment Procedures

\section{Introduction}

The study of the traffic psychology and behavior is in its infancy. There are studies related to various types of driving (dangerous driving, risky driving, aggressive driving and distracted driving) and to various psychological, demographic, gender factors affecting road safety. The use of mobile phones while driving is one of the significant reasons for distracted driving. A significant number of studies have shown that the use of a mobile phone impairs driving quality (Violanti \& Marshall, 1996; Violanti, 1999; Lamble, Rajalin, \& Summala, 2002; McEvoy, Stevenson \& Woodward, 2006), increases the risk of accidents (Violanti, 1999; Bener, Crundall, Özkan \& Lajunen, 2010), increases the reaction time of the driver (Patten, Kircher, Östlund, \& Nilsson, 2004), has a negative influence on driving style (Bener, Lajunen, Ozkan, \& Haigney, 2006), reduces driving performance (Treffner $\&$ Barrett , 2004) and diminishes driver's attention (Amado \& Ulupinar, 2005).

Scientists note that, in addition to the importance of the above factors, the prospect of scientific research should be aimed at studying the intentions and attitudes towards the use the mobile phone while driving (Lipovac, Đerić, Tešić, Andrić, \& Maric, 2017).

Stressing the importance of the study of attitudes towards risky and safe driving on the roads is attributable to the fact that they are able to influence the subject's intention and subsequent behavior. In line with modern views, attitudes provide a final assessment of the target object and are often assumed to follow the specific beliefs, emotions and past behavior associated with this object (APA, 2009). Scientists in the field of social cognition understand the attitudes to be the integration of cognitive and emotional assessments of an object that can change in strength (Olson \& Kendrick, 2008). The process of actualization of attitudes can require volitional efforts and be conscious, controlled or, conversely, spontaneous, unconscious and automatic. In the first case we are talking about explicit attitudes, in the second, about implicit ones. Implicit attitudes are evaluative tendencies that can influence judgments and behavior, even though the subject is not aware of it. (Plotka, Igonin, \& Blumenau, 2016). Explicit attitudes are understood as a product of, basically, propositional, logical processes, and implicit ones are the result of associative processes (Plotka et al., 2016). There are two approaches in cognitive psychology aimed at studying the processes of cognitive processing of information. The essence of the first approach is that it is based on studies of selective attention (Posner \& Snyder, 1975; Shiffrin \& Schneider, 1977), and the second - on implicit memory processes (Plotka et al., 2016). Based on the first approach, Fazio defines attitude as "an association in memory between a given object and a given summary evaluation of the object" 
(Fazio, 1995: p. 247) In the frameworks of the second approach Greenwald defines the implicit attitude as "introspectively unidentified (or inaccurately unidentified) traces of past experience that mediate favorable or unfavorable feeling, thought, or action toward social objects” (Greenwald, \& Banaji, 1995: p. 8)

Modern implicit measurements are based on measuring the reaction time (RT) of participants when they perform various tasks and their attention is focused on the fulfillment of these tasks, rather than on the object of attitudes (Rudman, 2011). Implicit measurements are indirect, automatic and unconscious (Petty, Fazio, \& Brinol, 2009). The classical Implicit Association Test (Greenwald, McGhee, \& Schwartz, 1998) and its varieties (Klauer, Voss, \& Stahl, 2011) are widely used. Explicit methods are direct, controlled and realized (Petty et al., 2009). These are measurements of deliberate assessments that come to mind after some reflection and are based on explicit knowledge about oneself and others (Petty et al., 2009).

It should be noted that there is a problem of both explicit and implicit measurements related to the study of attitudes towards the use of mobile phones.

Most researchers study explicit attitudes towards the use of mobile phones while driving, using self-assesment procedures (Ronggang, Changxu, Rau, \& Zhang, 2009; White, Hyde, Walsh, \& Watson, 2010; Nemme \& White, 2010; Ronggang, Rau, Zhang, \& Zhuang, 2012; Yannis, Theofilatos, \& Marinou, 2015; Lipovac et al, 2017). Studies of driving attitudes using implicit methodology were mainly focused on the study of attitudes towards speed infringement, attitudes towards the use of seat belts, attitudes towards driving in a state of intoxication, attitudes towards fatigue driving (Fernandes, Hatfield, \& Job, 2006; Hatfield, Fernandes, Faunce, \& Job, 2008), attitudes towards risky and safe driving (Martinussen, Sumhovd, Muller, \& Siebler, 2015), attitudes towards using motorcycle helmets during motorcycle driving (Ledesma, Tosi, Poo, Montes, \& López, 2015) and were focused on the study of role of implicit attitudes on road safety behaviors. Hatfield at al. (2008) made an important contribution to the development of implicit tools by studying attitudes towards speeding. The Implicit Association Test (IAT) developed by the researchers, turned out to be valid enough. Another group of researchers (Fernandes et al., 2006) studied the effect of predictive capabilities of demographic factors, personal characteristics and driver attitudes towards various types of driving using the Implicit Association Tests appropriate to each type of driving. The scientific finding was that it was attitudes that were the strongest predictors for all four kinds of risky driving and pointed to specificity for various types of driving. Martinussen at al. (2015) studying implicit attitudes towards risky/safe driving using a specific experimental procedure (GNAT) concluded that implicit attitudes towards risky and safe driving can be separate constructs and, accordingly, stem from different 
cognitive processes. Summing up the results of the above, we can state a shortage of studies aimed at developing tools (mostly implicit) designed to measure implicit attitudes towards different forms of driving. Analysis of the relevant literature showed that the study of attitudes towards the use of mobile phones while driving using implicit and explicit measurements are single, which led to the objectives of this research. For this purpose, two experimental procedures were developed for the measurement of attitudes towards the use of mobile phones while driving on the basis of the classical IAT and the self-concept IAT.

Research aim is the study of attitudes towards the use of mobile phones while driving with the use of Implicit Association Tests and self-assessment procedures.

\section{The Research questions are:}

1. Is there a relationship between measurement results of attitudes towards the use of mobile phones while driving using the IAT experimental procedures and the self-concept IAT?

2. Is there a relationship between measurement results of attitudes towards the use of mobile phones while driving, obtained with the help of experimental IAT procedures and the self-concept IAT with measurement results using the self-assessment procedure?

3. Is there a relationship between measurement results of attitudes towards the use of mobile phones while driving, measured using the IAT experimental procedure and the self-concept IAT and personal factors?

4. Is there a relationship between measurement results of attitudes towards the use of mobile phones while driving, measured by the selfassessment procedure and personal factors?

5. Which of the experimental IAT or self-concept IAT procedures show a larger size of the observed implicit effect of using mobile phones while driving?

\section{Method}

Participants: 69 drivers in the city of Riga with B Category drivers licenses, 27 females and 42 males, aged 21-59, $M d n=42$ years old.

\section{Implicit methods}

The experimental procedure of the Implicit Association Test (IAT) was developed on the basis of the classical two-categorical IAT (Greenwald, McGhee, \& Schwartz, 1998); Target categories were: visual stimuli - 12 pictures depicting people using a mobile phone while driving and 10 pictures depicting people who do not use the mobile phone while driving. When selecting pictures, their similarities in size, visual complexity and clarity of the image (absence of 
strangers, absence of celebrities, visibility of the road and the movement of the car) were taken into account. The target attributes were words with strong affective meaning. Positive words: attentive, disciplined, responsible, reasonable, safe, observant, pleasure, calm, prudent, concentrated and cautious; and negative words: absent-minded, light-minded, irresponsible, frivolous, dangerous, neglectful, disgusted, careless, anxious, reckless, distracted and risky. Preliminary, 80 drivers were interviewed in order to select the target attributes. The task of the participant was to describe the characteristics of the driver, who uses the mobile phone while driving with three adjectives. Next, the most common words were analyzed. As a result, 12 words were selected and antonyms were selected for them.

The experimental procedure of the self-concept Implicit Association Test was developed on the basis of the two-category self-concept IAT (Greenwald \& Farnham, 2000).

Target categories are: "I" and "Others". The target category "I" was represented by verbal stimuli I, my, me, mine and self; the target category "Others" was represented by verbal stimuli they, their, theirs, them and other.

Target attributes are: visual stimuli - pictures depicting the driver using and not using the mobile phone while driving, related to the target categories "Using the phone" and "Not using the phone", similar to the first IAT.

\section{Explicit methods:}

The self-assessment questionnaire "The Scale of Measuring Attitudes towards the Use of Mobile Phones while Driving” developed by the research authors and containing 31 statements, measuring the attitude towards using mobile phones while driving. Cronbach's Alpha is $\alpha=.93$.

The cross-cultural questionnaire of personal traits "ZKPQ-50-CC" (Aluja, Rossier, García, Angleitner, Kuhlman, \& Zuckerman, 2006), for which the primary linguistic adaptation was carried out. Cronbach's Alpha is $\alpha=.78$. The questionnaire consists of 50 statements that characterize the behavior of a person in typical life situations, in which his/her personality traits are most clearly manifested. The methodology includes the following scales: Impulsive Sensation Seeking, Sociability, Activity, Aggression-Hostility, Neuroticism-Anxiety. "Neuroticism-anxiety: measures anxiety, fear, general emotionality, psychasthenia, and inhibition of aggression. The factor is also associated with obsessive indecisiveness, lack of self-confidence, and sensitivity to criticism. Aggression-hostility vs. social desirability: measures aggression, hostility, anger, lack of inhibitory control, and low social desirability. The factor is associated with rudeness, thoughtless and antisocial behaviour, vengefulness, quick temper and impatience. Impulsive sensation-seeking: measures low socialisation, and high psychoticism, impulsivity, and sensation-seeking. The impulsivity items assess lack of planfulness and a tendency to act without thinking. The sensation seeking 
Vinogradova et al., 2018. Study of Attitudes Towards the Use of Mobile Phones While Driving with Implicit Association Tests and Self-Assessment Procedures

items describe a liking for thrills and excitement, novelty and variety, and unpredictable situations and friends. Sociability: measures affiliation, social participation, extraversion. Assesses liking for big parties and interactions with many people, as well as a dislike of isolation in sociable people versus a liking for the same in unsociable people. Activity: measures energetic behavior and persistence. This factor is associated with need to keep active and feelings of restlessness when there is nothing to do" (Zuckerman, 2002: pp. 382-383).

Apparatus: Certified licensed software E-Prime 2®.

Procedure of the research

IAT measure. Performance of the IAT took an average of 10 to 20 minutes. Participants' reaction time (RT) was registered. Each stage was preceded by a set of instructions concerning the dimensions of the categorization task and the appropriate key responses. Instructions were written in black letters on a white background and located in the center of the monitor screen. Before the start of the experiment, on a computer monitor a participant was given general instructions and specific instructions before each of the blocks (tasks). Each word or picture was presented in the screen center in random order. The task of the participants was the differentiation of presented stimuli. The IAT combined the verbal and visual stimulus. The stimulus word or picture displayed on the screen without auditory accompaniment and remained on the screen until the pressing a key of the participant (Table 1). The RT for each trail was recorded as the time interval between the onset of stimulus presentation and pressing the correct key. Words and pictures were selected randomly without replacement.

Table 1 IAT for mobile phone use while driving

\begin{tabular}{|c|c|c|c|}
\hline Block & Test & Function & Procedure \\
\hline 1 & 24 & Training block & "Using the phone" vs "Not using the phone" \\
\hline 2 & 24 & Training block & “Bad” vs “Good” \\
\hline 3 & 24 & $\begin{array}{l}\text { Critical block for } \\
\text { analysis }\end{array}$ & $\begin{array}{l}\text { "Using the phone" + "Bad" vs "Not using the } \\
\text { phone" + "Good" }\end{array}$ \\
\hline 4 & 48 & $\begin{array}{l}\text { Critical block for } \\
\text { analysis }\end{array}$ & $\begin{array}{l}\text { "Using the phone" + "Bad" vs "Not using the } \\
\text { phone" + "Good" }\end{array}$ \\
\hline 5 & 24 & Training block & "Not using the phone" vs "Using the phone" \\
\hline 6 & 24 & $\begin{array}{l}\text { Critical block for } \\
\text { analysis }\end{array}$ & $\begin{array}{l}\text { "Not using the phone" + "Bad" vs "Using the } \\
\text { phone" + "Good" }\end{array}$ \\
\hline 7 & 48 & $\begin{array}{l}\text { Critical block for } \\
\text { analysis }\end{array}$ & $\begin{array}{l}\text { "Not using the phone" + "Bad" vs "Using the } \\
\text { phone" + "Good" }\end{array}$ \\
\hline
\end{tabular}

Self-concept IAT measure. The procedure was similar to the IAT measure (Table 2). 
Table 2 Self-concept IAT for mobile phone use while driving

\begin{tabular}{|c|c|c|c|}
\hline Block & Test & Function & Procedure \\
\hline 1 & 20 & Training block & "Others" vs "I" \\
\hline 2 & 20 & Training block & "Using the phone" vs "Not using the phone" \\
\hline 3 & 20 & $\begin{array}{l}\text { Critical block for } \\
\text { analysis }\end{array}$ & $\begin{array}{l}\text { "Using the phone" + "Others" vs "Not using } \\
\text { the phone" + "I" }\end{array}$ \\
\hline 4 & 40 & $\begin{array}{l}\text { Critical block for } \\
\text { analysis }\end{array}$ & $\begin{array}{l}\text { "Using the phone" + "Others" vs "Not using } \\
\text { the phone" + "I" }\end{array}$ \\
\hline 5 & 20 & Training block & "I" vs “Others" \\
\hline 6 & 20 & $\begin{array}{l}\text { Critical block for } \\
\text { analysis }\end{array}$ & $\begin{array}{l}\text { "I" + "Using the phone" vs "Others" + "Not } \\
\text { using the phone" }\end{array}$ \\
\hline 7 & 40 & $\begin{array}{l}\text { Critical block for } \\
\text { analysis }\end{array}$ & $\begin{array}{l}\text { "I" + "Using the phone" vs "Others" + "Not } \\
\text { using the phone" }\end{array}$ \\
\hline
\end{tabular}

Each participant of the experimental study passed through two experimental procedures: the IAT and self-concept IAT. For the purity of the experiment, half of the participants had to undergo first the experimental procedure of the IAT (on the computer), undergo the experimental procedure of the self-concept-IAT (on the computer) in a couple of days after the first experiment and fill in explicit questionnaires. The second half of the participants were first asked to undergo the experimental procedure of the self-concept IAT (on the computer), undergo the experimental procedure of the IAT (on a computer) in a couple of days after the first experiment and fill in explicit questionnaires.

\section{Results}

Variables: $D, D(S)$ - are the effect sizes, obtained as a result of measurements using the IAT and the self-concept of the IAT, respectively.

$D \geq 0.15$ - the effect size of implicit preference for not using a mobile phone while driving obtained by IAT,

$D \leq-0.15$ - the effect size of implicit preference for using a mobile phone while driving, obtained by IAT.

$D(S) \geq 0.15$ - the effect size of implicit associations of perceiving oneself as a not using the mobile phone while driving, obtained by the self-concept IAT,

$D(S) \leq-0.15$ - the effect size of implicit associations of perceiving oneself as an using the mobile phone while driving, obtained by the self-concept IAT,

Attitude - is a variable measured using the self-evaluation procedure "Scale of measuring attitudes towards the use of mobile phones while driving". The quarters are: $Q_{1}=56, Q_{3}=85$. Variable values less than $Q_{1}$ indicate the high explicit preference for using the mobile phone while driving. Values greater than $Q_{3}$ - the low explicit preference. 
Vinogradova et al., 2018. Study of Attitudes Towards the Use of Mobile Phones While Driving with Implicit Association Tests and Self-Assessment Procedures

Personal factors: ImpSS - Impulsive Sensation Seeking; $N$-Anx Neuroticism-Anxiety; Agg-Host - Aggression-Hostility; Act - Activity; Sy Sociability.

For variables - personal factors, the division into groups by levels of severity was performed by quartiles: for values greater than $Q_{3}$ - high level, less than $Q_{1}$ low level.

Statistical methods. The analysis of the initial data showed that for the study it is possible to apply both parametric and nonparametric statistics. To answer all four research questions the Pearson's $(r)$ and Spearman's $\left(r_{s}\right)$ correlation coefficients were used. These correlation coefficients are indexes of the effect size. At their values effect sizes are: .1 - small, . 3 - medium, .5 - large (Ellis, 2010, p.41).

The first research question. In the general sample, no correlation between the results and measurements using the IAT and the self-concept of IAT was found. A positive correlation was found in the group of participants with a high level of neuroticism-anxiety: $r_{S}(25)=.59, p=.002$. The effect size is large.

The second research question. The correlation coefficients of the variables $D$ and Attitude, as well as $D(S)$ - and Attitude are shown in Table 3.

Table 3 Correlation coefficients between the results of IAT procedures $(D, D(S))$ and the self-evaluation procedure "Scale of measuring attitudes towards the use of mobile phones while driving” (Attitude)

\begin{tabular}{lccc}
\hline \multicolumn{1}{c}{ Variables } & Group & $\begin{array}{c}\text { Correlation } \\
\text { coefficient }\end{array}$ & $\begin{array}{c}\text { Effect } \\
\text { size }\end{array}$ \\
\hline Attitude $-D$ & Agg-Host low & $r_{S}(22)=-.50, p=.018$. & Large \\
Attitude $-D$ & Experience more 24 years & $r_{S}(19)=-.56, p=.013$. & Large \\
Attitude $-D(S)$ & Act low & $r_{S}(17)=.54, p=.024$ & Large \\
Attitude $-D(S)$ & Agg-Host high & $r(20)=.52, p=.019$ & Large \\
Attitude $-D(S)$ & Age 33-46 years & $r_{S}(33)=.36, p=.042$ & Medium \\
\hline $\begin{array}{l}\text { Note. Agg-Host - Aggression-Hostility; Act - Activity. } D, D(S)-\text { the effect sizes, of IAT and self- } \\
\text { concept IAT respectively. }\end{array}$
\end{tabular}


Table 4 Correlation coefficients for the results of measuring implicit attitudes towards the use of mobile phones while driving using the IAT and personal factors obtained using the explicit methodology of ZKPQ-50-CCVariable 1

\begin{tabular}{|c|c|c|c|c|}
\hline & Variable 2 & Group & $\begin{array}{c}\text { Correlation } \\
\text { coefficient }\end{array}$ & Effect size \\
\hline $\begin{array}{l}\mathbb{S} \\
a^{\circ}\end{array}$ & $\begin{array}{l}\text { ImpSS } \\
\text { ImpSS } \\
\text { ImpSS } \\
\text { N-Anx } \\
N-A n x \\
N-A n x \\
N-A n x \\
N-A n x \\
\text { Agg-Host } \\
\text { Agg-Host } \\
\text { Act } \\
\text { Act }\end{array}$ & $\begin{array}{l}21 \text { - } 32 \text { years } \\
\text { Sy medium } \\
D \text { no effect } \\
\text { Attitude no effect } \\
\text { Men } \\
\text { 33-46 years } \\
\text { Act high } \\
\text { Sy high } \\
21 \text { - } 32 \text { years } \\
\text { Agg-Host high } \\
\text { Act high } \\
\text { Act low }\end{array}$ & $\begin{array}{l}r_{S}(18)=-.54, p=.002 \\
r_{S}(21)=-.42, p=.057 \\
r_{S}(14)=-.75, p=.002 \\
r_{S}(34)=-.36, p=.039 \\
r_{S}(42)=-.33, p=.025 \\
r_{S}(33)=-.47, p=.005 \\
r(23)=-.48, p=.020 \\
r_{S}(27)=-.42, p=.030 \\
r(18)=-.49, p=.039 \\
r(20)=-.52, p=.019 \\
r_{S}(23)=-.55, p=.007 \\
r(17)=-.62, p=.009\end{array}$ & $\begin{array}{l}\text { Large } \\
\text { Medium } \\
\text { Large } \\
\text { Medium } \\
\text { Medium } \\
\text { Large } \\
\text { Large } \\
\text { Medium } \\
\text { Large } \\
\text { Large } \\
\text { Large } \\
\text { Large }\end{array}$ \\
\hline 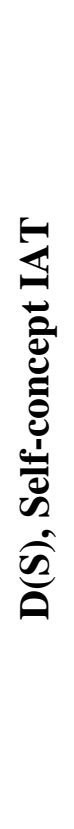 & $\begin{array}{l}\text { Act } \\
\text { Act } \\
\text { N-Anx } \\
\text { N-Anx } \\
\text { N-Anx } \\
\text { Agg-Host } \\
\text { Agg-Host } \\
\text { Agg-Host } \\
\text { ImpSS } \\
\text { Sy } \\
\text { Sy } \\
\text { Sy }\end{array}$ & $\begin{array}{l}\text { ImpSS low } \\
47-59 \text { years } \\
\text { Act high } \\
\text { Sy high } \\
\text { Sy medium } \\
\text { Women } \\
\text { Attitude: phone } \\
\text { Experience till } 9 \text { years } \\
N \text {-Anx medium } \\
N \text {-Anx high } \\
\text { Experience till } 9 \text { years } \\
N \text {-Anx medium }\end{array}$ & $\begin{array}{l}r_{S}(18)=-.47, p=.050 \\
r_{S}(18)=-.48, p=.046 \\
r_{S}(23)=-.45, p=.030 \\
r_{S}(27)=-.40, p=.038 \\
r_{S}(21)=.51, p=.020 \\
r_{S}(27)=-.39, p=.047 \\
r_{S}(17)=-.59, p=.012 \\
r_{S}(19)=-.62, p=.004 \\
r_{S}(16)=-.58, p=.019 \\
r_{S}(25)=-.43, p=.030 \\
r_{(19)}=-.52, p=.023 \\
r_{S}(16)=-.59, p=.017\end{array}$ & $\begin{array}{l}\text { Large } \\
\text { Large } \\
\text { Medium } \\
\text { Medium } \\
\text { Large } \\
\text { Medium } \\
\text { Large } \\
\text { Large } \\
\text { Large } \\
\text { Medium } \\
\text { Large } \\
\text { Large }\end{array}$ \\
\hline
\end{tabular}

Note. ImpSS - Impulsive Sensation Seeking; N-Anx - Neuroticism-Anxiety; Agg-Host - AggressionHostility; Act - Activity; Sy - Sociability 
Vinogradova et al., 2018. Study of Attitudes Towards the Use of Mobile Phones While Driving with Implicit Association Tests and Self-Assessment Procedures

The third research question. The correlation coefficients of the variables $D$ and $D(S)$ - the sizes of the effects obtained as a result of the measurement using the IAT and the self-concept IAT, respectively, with personality factors are shown in Table 4.

The forth research question. The coefficients of correlation between the Attitude variable measured using the self-evaluation procedure "Scale of measuring attitudes towards the use of mobile phones while driving" and personal factors are shown in Table 5.

Table 5 Correlation coefficients for measurement results of explicit attitudes towards the use of mobile phones while driving with the self-evaluation procedure "Scale of measuring attitudes towards the use of mobile phones while driving" and personal factors obtained using the explicit methodology of ZKPQ-50-CC

\begin{tabular}{|c|c|l|l|}
\hline Personal factor & Group & $\begin{array}{c}\text { Correlation } \\
\text { coefficient }\end{array}$ & \multicolumn{1}{c|}{$\begin{array}{c}\text { Effect } \\
\text { size }\end{array}$} \\
\hline Activity & $47-59$ years & $r_{S}(18)=.49, p=.041$ & Large \\
Activity & Agg-Host medium & $r_{S}(27)=-.46, p=.017$ & Large \\
Activity & Sy high & $r(27)=.46, p=.015$ & Large \\
Activity & Act high & $r(23)=.48, p=.022$ & Large \\
Activity & ImpSS high & $r S(23)=.57, p=.004$ & Large \\
Activity & $D(S)$ :I use” & $r_{S}(13)=.60, p=.032$ & Large \\
Aggression-Hostility & $D(S):$ "I don't use" & $r(35)=.34, p=.046$ & Medium \\
Aggression-Hostility & Sy low & $r(21)=.43, p=.053$ & Medium \\
Aggression-Hostility & $21-32$ years & $r_{S}(18)=.53, p=.024$ & Large \\
Aggression-Hostility & $N$-Anx medium & $r_{S}(16)=-.57, p=.022$ & Large \\
\hline
\end{tabular}

Note. ImpSS - Impulsive Sensation Seeking; N-Anx - Neuroticism-Anxiety; Agg-Host - AggressionHostility; Act - Activity; Sy - Sociability

The fifth research question. To answer the fifth research question Wilcoxon Signed Ranks Z-Test for paired samples was used. It revealed, that effect sizes, detected by IAT - the levels of $D(M d n=0.45)$ significantly higher than effect sizes, revealed with the help of the self-concept IAT - the levels of $D(S)(M d n=0.45)$ :

$Z=3.56, p<.001, r=.30$. The effect size for Wilcoxon's test is medium.

Note. $r$ - is the effect size for Wilcoxon's test: $r=|Z| / \sqrt{N}, N$ - is the number of observations (Field, 2009: p. 558). Effect size: .1 - small, . 3 - medium .5 large. 


\section{Discussion and Conclusions}

As a result of the research, its aim was implemented and the key results were presented; answers to research questions were received.

The theoretical analysis of the literature devoted to the problem under research has shown that among the many factors influencing dangerous/safe driving, the leading and insufficiently researched attitude is the attitude towards using mobile phones. The scarcity of the tools is largely presented with implicit measurements. In this connection, two experimental procedures were developed by the authors. The first procedure is an experimental procedure based on the twocategory IAT (Greenwald et al., 1998) that measures relationships between target categories (visual stimuli depicting the use/non-use of mobile phones while driving) and evaluating attributes (verbal stimuli) and negative valence. The second procedure is the experimental procedure (self-concept IAT), based on the two-category IAT (Greenwald \& Farnham, 2000) that measures implicit relationships between the target categories of I/Others and the perception of oneself as observing (not using the mobile phone while driving) / neglecting (using the mobile phone while driving) the traffic regulations. Also, the research authors developed the self-assessing procedure "Scale of Measuring Attitudes towards the Use of Mobile Phones while Driving," aimed at measuring explicit attitudes towards driving.

The answer to the first research question showed that there was no correlation between the results of the IAT and self-concept IAT measurements the effect sizes - by the total sample. However, in the group of participants with a high level of neuroticism-anxiety, a positive correlation was revealed. The size of the found effect is large. In this group of participants, the implicit preference for using the mobile phone while driving (IAT) corresponds to implicit associations of perceiving oneself as using the mobile phone while driving (self-concept IAT) and vice versa. Thus, in the group of participants with the high level of neuroticism-anxiety, a good consistency of the measurement results obtained by the experimental procedures of the IAT and the self-concept IAT is established.

Answering the second research question, it was found that the presence of the correspondence between the results of implicit measurements is associated with personality traits - aggression-hostility and with age.

For participants with the low level of aggression-hostility, the effect of the implicit preference of "not using a mobile phone while driving" (IAT) corresponds to the explicit attitude towards using mobile phones while driving and vice versa. (Table 3). Thus, for participants with the low level of aggressionhostility there is a inconsistency of the measurement results, using the IAT and the self-assessment procedure. The same inconsistency of the results of the IAT 
Vinogradova et al., 2018. Study of Attitudes Towards the Use of Mobile Phones While Driving with Implicit Association Tests and Self-Assessment Procedures

and an explicit procedure is observed for drivers with experience more than 24 years.

In the group of drivers with the high level of aggression-hostility, as well as in the group of drivers with the low level of activity, as well as in the group of drivers aged from 33 to 46, the effect of implicit associations of perception of themselves as using mobile phones while driving, measured with self-concept IAT, corresponds to the explicit positive attitude towards using mobile phone while driving and vice versa (Table 3). In these groups of drivers a good correspondence of the results of measurements with the self-concept IAT and selfreport procedure was established.

The answer to the third research question was the discovery of a relationship between measurement results of attitudes towards the use of mobile phones while driving, measured using the IAT experimental procedure and the self-concept IAT and personal factors (Table 4).

The implicit preference for using mobile phones while driving (the result of the IAT) is characteristic of the participants in the research, such as:

- with a high level of impulsive sensation seeking (in the groups: participants aged 21 to 32, participants with medium level of sociability, participants who did not show the effect of any implicit preference in performing the IAT);

- $\quad$ with a high level of neuroticism-anxiety (in the groups: participants men, participants aged 33 to 46, participants with high activity, participants with high sociability and participants who did not show the effect of any explicit preference);

- $\quad$ with a high level of aggressiveness-hostility (in the group: participants aged 21 to 32);

- $\quad$ with a high level of activity.

Implicit associations of perceptions of oneself as using the mobile phone while driving (the result of the self-concept IAT) are characteristic of the participants in the research, such as:

- $\quad$ with a high level of activity (in the groups: participants with a low level of impulsive sensation seeking, participants aged 47 to 59);

- $\quad$ with a high level of neuroticism-anxiety (in the groups: participants with a high level of activity and participants with high level of sociability);

- with a low level of neuroticism-anxiety from the participants with medium level of sociability;

- with a high level of aggression-hostility (in the groups: women, participants with the experience of driving till 9 years, participants who showed the explicit preference to mobile phone while driving); 
- $\quad$ with a high level of impulsive sensation seeking from the participants with medium level of neuroticism-anxiety;

- $\quad$ with a high level of sociability (in the groups: participants with a high level of neuroticism-anxiety, participants with the experience of driving till 9 years, and participants with a medium level of neuroticismanxiety).

Responding to the fourth research question, a relationship was found between the measurement results of attitudes towards the use of mobile phones while driving, measured by the self-assessment procedure and some personal factors (Table 5).

The explicit preference for using the mobile phone while driving is characteristic of the participants in the research, such as:

- $\quad$ with a high level of aggression-hostility (in the groups: participants with implicit associations of perceiving oneself as a not using the mobile phone while driving, obtained by the self-concept IAT, participants with a low level of sociability, participants aged from 21 to 32, participants with a medium level of neuroticism-anxiety);

- $\quad$ with a low level of activity (in the groups: participants aged from 47 to 59 years, participants with a high level of sociability, participants with a high level of activity, participants with a high level of impulsive sensation seeking and participants with implicit associations of perceiving oneself as an using the mobile phone while driving, obtained by the self-concept IAT);

- $\quad$ with a high level of activity in the groups of participants with a medium level of aggression-hostility).

The fifth research question related to the point whether the experimental IAT or the self-concept IAT procedures show a larger size of the observed implicit effect of using mobile phones while driving. It was shown that the effect sizes of implicit preferences detected by IAT statistically significantly exceed the effect sizes of implicit associations detected by the self-concept IAT. The size of the resulting excess effect is average.

The limitations of the research relate to the fact that the consistency of measurement results obtained by the IAT experimental procedures and the selfconcept IAT refers to only a part of the sample. In this research, it was not possible to use random sampling.

Research perspectives are aimed at developing new experimental procedures for both the Implicit Association Test and the unconscious emotional priming in relation to attitudes towards driving. 
Vinogradova et al., 2018. Study of Attitudes Towards the Use of Mobile Phones While Driving with Implicit Association Tests and Self-Assessment Procedures

It is necessary to conduct further research aimed at both improving the tools used and creating a sample that will allow us to find additional variables under which the consistency of measurement results could be achieved.

The results of the research can be used by various psychological services in the development of psycho-correction and educational programs. The patterns identified in the research can be taken into account in the development of preventive measures and strategies aimed at improving road safety, as well as corrective programs to form attitudes towards safe driving.

\section{References}

Aluja, A., Rossier, J., Garcia, L. F., Angleitner, A., Kuhlman, M., \& Zuckerman, M. (2006). A cross-cultural shortened form of the ZKPQ (ZKPQ-50-cc) adapted to English, French, German, and Spanish languages. Personality and Individual Differences, 41, 619-628.

Amado, S., \& Ulupinar, P. (2005). The effects of conversation on attention and peripheral detection: Is talking with a passenger and talking on the cell phone different? Transportation Research Part F, 8, 383-395. http://dx.doi.org/10.1016/j.trf.2005.05.001.

American Psychological Association (2009). APA concise dictionary of psychology. Washington, DC: American Psychological Association.

Bener, A., Lajunen, T., Ozkan, T., \& Haigney, D. (2006). The effect of mobile phone use on driving style and driving skills. IJC Crash, 11 (5), 459-465. Retrieved from http://dx.doi.org/10.1533/ijcr.2005.0116,

http://www.salimandsalimah.org/doc_uploads/research/Theeffectofmobilephoneuse.pdf.

Bener, A., Crundall, D., Ozkan, T., \& Lajunen, T. (2010). Mobile phone use while driving: A major public health problem in an Arabian society, State of Qatarmobile phone use and the risk of motor vehicle crashes. Journal Public Health, 18, 123-129. Retrieved from http://dx.doi.org/10.1007/s10389-009-0286-1.

Ellis, P. D. (2010). The essential guide to effect sizes. Statistical power, meta-analysis, and the interpretation of research results. Cambridge: University Press.

Fazio, R. H., Chen, J., McDonel, E. C., \& Sher-man, S. J. (1982). Attitude accessibility, attitude-behavior consistency and the strength of the object-evaluation association. Journal of Experimental Social Psychology, 18, 339-357.

Fazio, R. H. (1995). Attitudes as object-evaluation associations: Determinants, consequences, and correlates of attitude accessibility. In R. E. Petty \& J. A. Krosnick (Eds.), Attitude strength: Antecedents and consequences (pp. 247-288). Mahwah, NJ: Erlbaum.

Fernandes, R. F., Hatfield, J., \& Job, R. F. S. (2006). Examination of different predictors of different risky driving behaviours in young NSW drivers. Final report for the Motor Accidents Authority of NSW, Australia. Retrieved from http://www.irmrc.unsw.edu.au/ documents/predictors\%20of\%20risky\% 20drving\%20report.pdf.

Field, A. (2009). Discovering statistics using SPSS (3rd Edition). Washington DC: Sage. Available at http://fac.ksu.edu.sa/sites/default/files/ktb_lktrwny_shml_fy_lhs.pdf.

Greenwald, A. G., \& Banaji, M. R. (1995). Implicit social cognition: Attitudes, self-esteem, and stereotypes. Psychological Review, 102, 4-27. doi:10.1037/0033-295X.102.1.4.

Greenwald, A. G., McGhee, D. E., \& Schwartz, J. L. K. (1998). Measuring individual differences in implicit cognition: The Implicit Association Test. Journal of Personality and Social Psychology, 74, 1464-1480. 
Greenwald, A. G., \& Farnham, S. D. (2000). Using the Implicit Association Test to measure self-esteem and self-concept. Journal of Personality and Social Psychology, 79, 10221038.

Hatfield, J., Fernandes, R., Faunce, G., \& Job, R. F. S. (2008). An implicit non-self-report measure of attitudes to speeding: Development and validation, Accident Analysis and Prevention, vol. 40, pp. 616 - 627 https://doi.org/10.1016/j.aap.2007.08.020.

Jacoby, L. L. \& Dallas, M. (1981). “On the Relationship between Autobiographical Memory and Perceptual Learning,” Journal of Experimental Psychology: General, 110, 306-340.

Klauer, K. C., Voss, A., \& Stahl, C. (2011). Cognitive methods in social psychology. New York: Guilford Press.

Lamble, D., Rajalin, S., \& Summala, H. (2002). Mobile phone use while driving: public opinions on restrictions. Transportation, 29 (3), 223-236. http://dx.doi.org/10.1023/ A:1015698129964. Retrieved from http:/virtual.vtt.fi/virtual/proj6/fits/impacts/ Lamble_2002_Mobile_phone_use_while_driving.pdf

Lipovac, K., Đerić, M., Tešić, M., Andrić, Z., \& Maric, B., (2017). Mobile phone use while driving-literary review. Transportation Research Part F: Traffic Psychology and Behaviour. 47, 132-142. 10.1016/j.trf.2017.04.015.

Martinussen, L. M., Sømhovd, M. J., Møller, M., \& Siebler, F. (2015). A go/no-go approach to uncovering implicit attitudes towards safe and risky driving. Transportation Research Part F, 30, 74-83.

Nemme, H. E., \& White, K. M. (2010). Texting while driving: Psychosocial influences on young people's texting intentions and behaviour. Accident Analysis and Prevention, 42, 1257-1265. Retrieved from http://dx.doi.org/10.1016/j.aap.2010.01.019.

McEvoy, S. P., Stevenson, M. R., \& Woodward, M. (2006). Phone use and crashes while driving: A representative survey of drivers in two Australian states. The Medical Journal of Australia, 185, 630-634 https://www.mja.com.au/system/files/issues/185_11_041206/ mce10252_fm.pdf.

Olson, M. A., \& Kendrick, R. V. (2008). Origins of attitudes. In W. Crano \& R. Prislin (Eds.), Attitudes and Persuasion. New York: Psychology Press.

Patten, C. J. D., Kircher, A., Östlund, J., \& Nilsson, L. (2004). Using mobile telephones: Cognitive workload and attention resource allocation. Accident Analysis and Prevention, 36, 341-350. Retrieved from http://dx.doi.org/10.1016/S0001-4575(03)00014-9.

Petty, R. E., Fazio, R. H., \& Briñol, P. (2009). Attitudes: Insights from the new wave of implicit measures (85-117). In B. Gawronski, F. Strack, \& G. V. Bodenhausen, Attitudes and cognitive consistency: the role associative and propositional processes. New York: Psychology Press.

Plotka, I., Igonin, D., \& Blumenau, N. (2016). Implicit Attitudes and Measurements: Effect of Context. International Business: Innovations, Psychology and Economics, 7 (2 (12)), 7 150. Retrieved from http://www.kuryba.lt/failai/zurnalai/2016_2.pdf.

Posner, M. I., \& Snyder, C. R. (1975). Attention and cognitive control. In R. L. Solso (Ed.), Information processing and cognition: The Loyola symposium. Hillsdale, NJ: Erlbaum.

Ronggang, Z., Changxu, W., Rau, P. L. P., \& Zhang, W. (2009). Young driver's learners intention to use a hand-held or hands-free mobile phone when driving. Transportation Research Part F, 12, 208-217. http://dx.doi.org/10.1016/j.trf.2008.11.003.

Ronggang, Z., Rau, P. L. P., Zhang, W., \& Zhuang, D. (2012). Mobile phone use while driving: Predicting drivers answering intentions and compensatory decisions. Safety Science, 50, 138-149. Retrieved from http://dx.doi.org/10.1016/j.ssci.2011.07.013. 
Vinogradova et al., 2018. Study of Attitudes Towards the Use of Mobile Phones While Driving with Implicit Association Tests and Self-Assessment Procedures

Rudman, L. A. (2011). Implicit measures for social and personality psychology. London: Sage. Schacter, D. L. (1987). Implicit memory: History and current status. Journal of Experimental Psychology: Learning, Memory, and Cognition, 13, 501-518.

Shiffrin, R. M., \& Schneider, W. (1977). Controlled and automatic human information processing: II. Perceptual learning, automatic attending and a general theory. Psychological Review, 84 (2), 127-190. http://dx.doi.org/10.1037/0033-295X.84.2.127

Treffner, P. J., \& Barrett, R. (2004). Hands-free mobile phone speech while driving degrades coordination and control. Transportation Research Part F, 229-246. Retrieved from http://dx.doi.org/10.1016/j.trf.2004.09.002.

Violanti, J. M., \& Marshall, J. R. (1996). Cellular phones and traffic accidents: An epidemiological approach. Accident Analysis and Prevention, 28 (2), 265-270. Retrieved from http://dx.doi.org/10.1016/0001-4575(95)00070-4.

Violanti, J. M. (1999). Cellular phones and fatal traffic collisions. Accident Analysis and Prevention, 30 (4), 519-524. Retrieved from http://dx.doi.org/10.1016/S00014575(97)00094-8

White, K. M., Hyde, M. K., Walsh, S. P., \& Watson, B. (2010). Mobile phone use while driving: An investigation of the beliefs influencing drivers hands-free and hand-held mobile phone use. Transportation Research, Part F, 13, 9-20. Retrieved from http://dx.doi.org/ 10.1016/j.trf.2009.09.004.

Yannis, G., Theofilatos, A., \& Marinou, P. (2015). Attitudes of Greek Drivers with Focus on Mobile Phone Use While Driving, Traffic Injury Prevention, 16 (8), 831-834. DOI: 10.1080/15389588.2015.1030737.

Zuckerman, M. (2002). "Zuckerman-Kuhlman Personality Questionnaire (ZKPQ): an alternative five-factorial model”. In B. de Raad \& M. Perugini (Eds), Big Five Assessment (pp. 377-396). Hogrefe \& Huber Publishers. 